\title{
Perda funcional e depressão em paciente com infecção protética por M. tuberculosis após artroplastia de joelho: um relato de caso
}

\author{
Eduardo M. Costa, ${ }^{1 *}$ Mariangela Perez, ${ }^{2}$ Alfredo M. Villardi, ${ }^{3}$ Roberto A. Lourenço ${ }^{4}$
}

\section{Resumo}

Paciente idosa com quadro de infecção em prótese de joelho esquerdo, apresentando limitação funcional por dor e grande dificuldade de locomoção. Concomitantemente, apresentava o humor deprimido. Foi iniciado tratamento para depressão com duloxetina visando também melhor controle da dor, obtendo-se boa resposta. Paciente realizou novo procedimento cirúrgico para retirada da prótese, sendo evidenciada em cultura do material infecção por Staphylococcus capitis e Complexo Mycobacterium tuberculosis. Após o tratamento com esquema rifampicina (150 mg), isoniazida (75 mg), pirazinamida (400 $\mathrm{mg}$ ) e etambutol (275 mg) por nove meses, a paciente evoluiu com melhora significativa da dor e com recuperação da funcionalidade. A colocação de nova prótese restabeleceu sua independência prévia.

Descritores: Depressão; Perda funcional; Infecção protética.

\section{Abstract \\ Functional loss and depression in M. tuberculosis prosthetic infection after knee arthroplasty: a case report}

Elderly patient, presenting infection in a left knee prosthesis. She presented functional limitation due to the pain, with great difficulty of mobility. In addition, her mood was depressed. Treatment for depression with duloxetine was initiated, aiming at better control of pain, obtaining a good response. Patient performed a new surgical procedure to remove the prosthesis, with evidence of infection by Staphylococcus capitis and Mycobacterium tuberculosis Complex in the culture. After treatment with a 9-month Rifampcine (150 mg), Isoniazid (75 mg), Pirazinamide (400 mg) and Etambutol (275 $\mathrm{mg}$ ) regimen, the patient progressed with significant pain improvement and functional recovery, with the placement of a new prosthesis, and reestablishing her independence.

Keywords: Depression; Functional loss; Prosthesis infection.
1. Serviço de Geriatria Professor Mario A. Sayeg. Policlínica Piquet Carneiro. Universidade do Estado do Rio de Janeiro. Rio de Janeiro, RJ, Brasil.

2. Laboratório de Pesquisa em Envelhecimento Humano. Faculdade de Ciências Médicas. Universidade do Estado do Rio de Janeiro. Rio de Janeiro, RJ, Brasil

3. Serviço de Ortopedia. Hospital São Vicente de Paulo. Rio de Janeiro, RJ, Brasil.

4. Departamento de Medicina Interna. Faculdade de Ciências Médicas. Universidade do Estado do Rio de Janeiro. Rio de Janeiro, RJ, Brasil.

*Endereço para correspondência: GeronLab-UERJ Av. Marechal Rondon, 381, 20 andar, Rio de Janeiro, RJ, Brasil. CEP: 20950-000. E-mail: eduardomagalhaes88@yahoo.com.br

Revista HUPE, Rio de Janeiro, 2017;16(1):61-63 doi: $10.12957 /$ rhupe.2017.33290 Recebido em 26/01/2018. Aprovado em 08/02/2018.

\section{Resumen}

Pérdida funcional y depresión en infección protésica por $M$. tuberculosis después de la artroplastia de rodilla: un relato de caso

Paciente anciana, presentando infección en prótesis de rodilla izquierda. Debido al dolor presentaba limitación funcional, con gran dificultad de locomoción. Además, presentaba el humor deprimido. Se inició tratamiento para la depresión con duloxetina buscando un mejor control del dolor, obteniéndose buena respuesta. La paciente realizó un nuevo procedimiento quirúrgico para retirar la prótesis, siendo evidenciada en cultivo del material infección por Staphylococcus capitis y Complejo Mycobacterium tuberculosis. Después del tratamiento con esquema Rifampcina (150 mg), Isoniazida (75 mg), Pirazinamida (400 mg) y Etambutol ( $275 \mathrm{mg}$ ) por nueve meses, la paciente evolucionó con una mejora importante del dolor y recuperación de la funcionalidad, siendo realizada la colocación de nueva prótesis y reestableciendo su independencia previa.

Palabras clave: Depresión; Perda funcional; Infección de prótesis. 


\section{Caso clínico}

\section{Introdução}

A osteoartrose de joelho é uma condição que gera grande impacto na vida social dos pacientes idosos, pois a dor intensa e as deformidades geradas pela lesão geralmente ocasionam perda da independência. Isolados do seu ciclo social e mais dependentes, estes idosos têm maior chance de desenvolver outras síndromes geriátricas, entre elas, a depressão. O tratamento mais indicado nos casos de dor intensa e limitação funcional é a artroplastia de joelho (ATJ). A finalidade deste procedimento é aliviar a dor, obter ganho funcional e corrigir deformidades. A ATJ consiste na substituição da articulação em seus segmentos femoral, tibial e patelar por próteses. ${ }^{1,4}$

Trata-se de uma cirurgia de grande porte que necessita de avaliação pré-operatória minuciosa e rigoroso acompanhamento pós-operatório. Algumas complicações, apesar de raras, são possíveis, como a infecção da prótese. O objetivo deste relato foi apresentar uma complicação rara por um patógeno pouco usual (Complexo M. tuberculosis) após a cirurgia de ATJ em uma paciente idosa.

\section{Caso clínico}

GSD, sexo feminino, 83 anos, natural da cidade do Rio de Janeiro, onde reside, viúva, auxiliar administrativa aposentada. Compareceu ao Ambulatório de Geriatria encaminhada pelo Serviço de Ortopedia, ambos de um hospital geral da rede particular no Rio de Janeiro, para avaliação de risco cirúrgico de revisão de artroplastia em joelho esquerdo. Queixava-se de dor e rubor no joelho esquerdo e referia dificuldade de locomoção.

A história da doença atual teve início dois anos antes (2013) da avaliação geriátrica, quando a paciente se submeteu à primeira artroplastia total do joelho esquerdo (ATJE). No ano seguinte, precisou de nova abordagem cirúrgica por deslocamento da prótese. Em janeiro de 2015, retornou ao Serviço de Ortopedia por piora da dor no joelho esquerdo e perda da funcionalidade pela dificuldade de locomoção. Ao exame clínico e complementar foram encontradas evidências de processo infeccioso na prótese, sendo indicada a retirada desta, com posterior substituição. Em março de 2015, procurou o Serviço de Geriatria de um hospital geral da rede particular no Rio de Janeiro para avaliação pré-operatória.

Na história patológica pregressa apresentava hipotireoidismo, hipertensão arterial sistêmica, osteopenia, uma internação por hemorragia digestiva após uso abusivo de anti-inflamatório e diverticulite, com cirurgia prévia. Estava em uso de levotiroxina $50 \mathrm{mcg} / \mathrm{dia}$, omeprazol $20 \mathrm{mg} / \mathrm{dia}$, anlodipino $5 \mathrm{mg} / \mathrm{dia}$, losartana $50 \mathrm{mg} / \mathrm{dia}$, sinvastatina $20 \mathrm{mg} /$ dia, ginko-biloba $80 \mathrm{mg} /$ dia e alprazolam $2 \mathrm{mg} / \mathrm{dia}$. O suporte social era adequado e a história familiar, sem antecedentes significativos.

Na avaliação geriátrica ampla pré-operatória evidenciou-se dependência funcional, depressão, incontinência urinária e polifarmácia. $O$ rastreio cognitivo e as avaliações nutricional e social não apresentaram alterações.

Como primeira intervenção geriátrica foi prescrita a duloxetina, com o objetivo antidepressivo e também para modulação da dor crônica do joelho. Iniciou-se com a dose de $30 \mathrm{mg}$ por duas semanas, progredindo para $60 \mathrm{mg}$. A paciente foi liberada para o procedimento cirúrgico com risco ASA II, após realização de exames e ajustes da prescrição.

Em junho de 2015 a idosa submeteu-se ao procedimento cirúrgico de revisão da prótese do joelho esquerdo. Foram coletados materiais (fragmento ósseo de tíbia e fêmur, cimento ósseo, cimento do canal sinovial, líquido sinovial, membrana sinovial e sinóvia) para culturas durante a cirurgia e foi colocado um espaçador. $\mathrm{Na}$ cultura do cimento ósseo foi evidenciada infecção por Staphylococcus capitise iniciado antibioticoterapia com sulfametaxozol e trimetropim na dose $160 \mathrm{mg} / 800 \mathrm{mg}$ de $12 / 12 \mathrm{~h}$ por três semanas. Posteriormente, o esquema foi trocado para daptomicina ( $4 \mathrm{mg} / \mathrm{kg}$ ), suspensa após a paciente apresentar farmacodermia, e rifampicina por seis semanas, seguindo a orientação do Serviço de Infectologia da unidade hospitalar. Durante a internação a paciente apresentou dois episódios de infecção do trato urinário, tratados com ertapenem $1 \mathrm{~g}$ ao dia (guiado por urinocultura).

A paciente evoluiu com melhora importante dos sinais inflamatórios em joelho e dos sintomas urinários. Recebeu alta hospitalar em julho de 2015 para seguimento ambulatorial. Em setembro de 2015, o restante do material enviado para cultura evidenciou, no cimento do canal sinovial e no fragmento ósseo da tíbia, o Complexo Mycobacterium tuberculosis. A paciente foi então encaminhada para o centro de referência em tratamento de doenças infectocontagiosas, onde foi iniciado o esquema com rifampcina (150 mg), isoniazida $(75 \mathrm{mg}$ ), pirazinamida $(400 \mathrm{mg}$ ) e etambutol (275 mg) em outubro de 2015, com previsão de duração do tratamento entre 6 e 9 meses. A paciente evoluiu com melhora clínica e laboratorial dos marcadores inflamatórios. 
Após completar o tratamento com rifampicina e isoniazida (prorrogado para 9 meses), o espaçador foi substituído pela nova prótese de joelho em junho de 2016. A paciente apresentou rotação da patela no pósoperatório e necessitou de nova abordagem cirúrgica, que foi bem-sucedida. $\mathrm{O}$ uso do antidepressivo no período foi mantido, com bom controle do humor e da dor no membro. Após a última correção cirúrgica, a paciente apresentou melhora significativa da mobilidade e foi liberada para deambular sem órtese, evidenciando maior independência funcional.

\section{Discussão}

Sabe-se que a gonoartrose com limitação funcional é uma queixa prevalente, tanto em consultas de ortopedia quanto de geriatria. O tratamento cirúrgico é uma opção terapêutica quando há falência da abordagem conservadora (analgesia e fisioterapia). Dentre as complicações da artroplastia de joelho, observa-se a infecção da prótese, cuja incidência, entretanto, é baixa, em torno de $1 \%$ nos grandes centros especializados. ${ }^{1}$ Nos Estados Unidos, o impacto financeiro causado por esta complicação chega a trezentos milhões de dólares por ano. ${ }^{1}$ A contaminação pode ocorrer por implantação direta no ato operatório, por via hematogênica ou reativação de focos quiescentes. Os microrganismos mais comuns envolvidos na infecção são bactérias Gram positivas, seguida das Gram negativas; as menos prevalentes nas artroplastias de joelhos infectadas são fungos e o bacilo da tuberculose. ${ }^{1} \mathrm{O}$ caso relatado mostra uma infeção pós artroplastia de joelho por dois microorganismos Staphylococcus aureus e Mycobacterium tuberculosis. Trata-se de uma complicação rara que apresenta desfecho favorável se identificada e tratada de forma rápida e adequada. ${ }^{2}$ Sabe-se que quanto maior o número de abordagens, maior a chance do paciente desenvolver infecção de prótese. ${ }^{3}$

Uma série de 10 casos avaliou a artroplastia total de joelho como tratamento para melhora funcional e redução de dor em pacientes com acometimento ósseo por tuberculose. Evidenciou-se que o procedimento apresentava bom resultado, quando aliado ao tratamento medicamentoso. ${ }^{8} \mathrm{O}$ tratamento medicamentoso da tuberculose óssea nesta série de casos teve duração de 12 a 18 meses. Tal abordagem foi utilizada devido à pouca penetração das drogas nos tecidos ósseo e fibroso..$^{8}$ Entretanto, alguns autores têm preconizado cursos mais curtos de tratamento ( 6 a 9 meses) contendo rifampicina, já que alguns estudos demonstraram resultados parecidos quando comparados aos tratamentos mais longos sem essa droga. ${ }^{9}$ As diretrizes do Centro de Controle de Doenças norte-americano (CDC) e da
Sociedade Brasileira de Pneumologia sobre tuberculose recomendam um tratamento por seis meses para todos os casos de tuberculose extrapulmonar, exceto os que acometam as meninges, que possuem um tratamento diferenciado dada sua alta morbimortalidade. ${ }^{10,11}$

A paciente apresentava algumas síndromes geriátricas desencadeadas pelo processo infeccioso, como limitação funcional e depressão. ${ }^{4}$ Após o início do tratamento para depressão com duloxetina, observou-se melhora significativa da dor e do humor, o que contribuiu para um melhor resultado no pós-operatório. Este antidepressivo, além de atuar no controle de dor neuropática, também apresenta eficácia quando usado para controle de dor osteoarticular. ${ }^{5-7}$ Observou-se, ainda, que após o início desta medicação a paciente recuperou parte da funcionalidade, passou a deambular sozinha, o que teve um impacto positivo na sua recuperação pós-operatória. Posteriormente realizou troca da prótese com maior recuperação da funcionalidade.

\section{Referências}

1. Lima ALLM, Pécora JR, Albuquerque RM, et al. Infecção pós-artoplastia total do joelho - Considerações e protocolo de tratamento. Acta Ortop Bras. 2004;12(4).

2. Marmor M, Parnes N, Dekel S. Tuberculosis infection complicating total knee arthroplasty. J Arthroplasty. 2004;19(3).

3. Fonseca F. Infection in knee arthroplasties. Rev Port Ort e Trauma. 2006;14(2).

4. Moraes EN, Marino MCA, Santos RR. Principais síndromes geriátricas. Rev Med Minas Gerais. 2010;20(1):54-66.

5. Brown JP, Boulay LJ. Clinical experience with duloxetine in the management of chronic musculoskeletal pain. A focus on osteoarthritis of the knee. Ther Adv Musculoskelet Dis. 2013;5(6):291-304

6. Wang ZY, Shi SY, Li SJ, et al. Efficacy and Safety of Duloxetine on Osteoarthritis Knee Pain: A Meta-Analysis of Randomized Controlled Trials. Pain Med. 2015;16:1373-85.

7. Smith HS, Smith EJ, Smith BR. Duloxetine in the management of chronic musculoskeletal pain. Clin IntervAging. 2016:11.

8. Habaxi KK, Wang L, Miao XG, et al. Total knee arthroplasty treatment of active tuberculosis of the knee: a review of 10 cases. Eur Rev Med Pharmacol Sci. 2014;18:3587-92.

9. Sebben AL, Dotta G, Oliveira AEL, et al. Tuberculose de articulação coxofemoral: a propósito de um caso. Rev Assoc Med Bras. 2012;58(1):15-7.

10. Nahid P, Dorman SE, Alipanah N, et al. Official American Thoracic Society/Centers for Disease Control and Prevention/ Infectious Diseases Society of America Clinical Practice Guidelines: Treatment of Drug-Susceptible Tuberculosis. Clin Infect Dis. 2016;63(7):147-95.

11. Comissão de Tuberculose da SBPT, Grupo de Trabalho das Diretrizes para Tuberculose da SBPT. III Diretrizes para Tuberculose da Sociedade Brasileira de Pneumologia e Tisiologia. J Bras Pneumol. 2009;35(10):1018-48. 